\title{
A Goal Based Cost-Benefit Analysis for Film Versus Filmless Radiology Departments
}

\author{
Steve Langer and Jihong Wang
}

\begin{abstract}
We describe the use of spreadsheet models to compare differential costs incurred in a film based versus a filmless radiology department. The spreadsheet allows a high degree of user customization to facilitate on-site cost analysis and informed purchasing decisions. Modeling institutions that perform 25, 50, and 105,000 diagnostic procedures per year, we find that break-even occurs near 50,000 exams. Plots further show the trends in annual hardware and staff costs as work loads increase.

Copyright 1996 by W.B. Saunders Company
\end{abstract}

KEY WORDS: picture archiving and communication system (PACS), radiology information system (RIS) film, cost analysis, network performance.

$\mathbf{T}$

THE ROAD from a film-based radiology department to an all digital one can be long and potholed. Before embarking on it, radiology practitioners must endeavor to map the path as completely as possible, not only to execute the journey, but to justify it at all from a cost or quality standpoint. Fortunately, the exercise of cost analysis, if done well, pays dividends in the form of a detailed implementation plan that can then be used to design the digital department.

A number of authors have performed cost analyses on digital radiology departments. ${ }^{1-3} \mathrm{By}$ relying on computer simulations, these authors were able to identify possible bottlenecks and performance limitations. However, for radiology planners without access to simulation software, such an analysis is difficult. Furthermore, without using numbers germane to a specific practice, a computer model developed elsewhere can only be of limited use. For instance: one author identifies the break-even number (beyond which digital systems are more cost competitive than film) at 60,000 procedures per year in 1995 using low speed (Ethernet or Token Ring) networks. ${ }^{2}$ However, price esti-

From the Department of Diagnostic Radiology, Mayo Clinic and Foundation, Rochester, $M N$

Address reprint requests to Steve Langer, PhD, the Department of Diagnostic Radialogy, Mayo Clinic and Foundation, 200 First St SW, Rochester, MN 55905.

Copyright $\circledast 1996$ by W.B. Saunders Company

0897-1889/96/0903-0002\$3.00/0 mates used in that report may no longer apply. It is our goal in this work to provide an comprehensible information flow architecture, which can be modeled with simple spreadsheets. We provide details of the spreadsheet use in the appendices, and an anonymous ftp site where the actual files may be acquired in Microsoft Excel format (Microsoft Corp, Redmond, WA) for Windows and Macintosh platforms.

\section{MATERIALS AND METHODS}

Our spreadsheets model both a film-based and all digital department. Only differential costs are modeled, that is, only equipment and staff that would be changed in each case. We assume that the institution being modeled uses either a totally electronic medical record (EMR) environment, or relies totally on film based information management. This caveat is reasonable because the full economies of picture archiving and communication-radiology information system (PACS-RIS) cannot be realized until a total EMR environment is implemented. The model begins with patient admittance and tracks the resulting events across departmental boundaries. Cooperation among radiology, IS, billing, scheduling, and other departments is necessary to accurately count all transactions. Once these data are in hand, it is possible to arrive at workload estimates. The interaction of workload with desired performance then determines staffing and equipment needs-and ultimately costs.

Workload. To compute workload, it is necessary to track all patient data transactions from admittance through exam scheduling, exam creation, interpretation, archiving, and postinterpretation exam release to other departments. The transactions can be classed into two major categories: patient initiated and care provider initiated

When the patient is admitted they are scheduled for various exams. At the heart of the EMR environment is a hospital information system (HIS) and scheduling system (SS). The HIS will be capable of displaying all patient information including lab results, textual reports, radiological images, as well as demographics, and insurance information. ${ }^{4}$ The HIS retrieves this information by interrogating the appropriate departmental information systems. Similarly, the SS interrogates departmental information systems when scheduling tests. Each department is responsible for managing its own internally generated information. The data flow among several departments could be shown as in Fig 1 (only data flow in the EMR environment is charted, as the film department would be analogous). The HIS and SS may be the same application, or cooperating programs on separate computer systems. In either case they occupy a central position from a logic standpoint and must share information. Every department's internal information sys- 


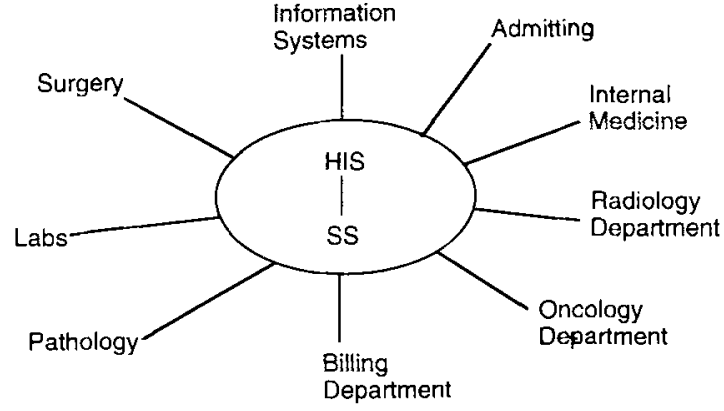

Fig 1. A data flow diagram showing the central role of the HIS and SS in coordinating the activities of the healthcare center.

tem must be accessible to the HIS-SS and be accounted for in computing the PACS-RIS workload

If the patient is scheduled for radiological tests a scheduler uses the SS to query the RIS for a time. Depending on the exam, the RIS may respond with a time but also inform the HIS-SS if the patient must be specially prepped for the exam. Furthermore, the RIS should be capable of informing the SS as to how long the exam should take so that other exams do not overlap in time. A detailed view of the transaction is shown in Fig 2.

On scheduling the exam, the RIS initiates a series of events to prepare for the patient's arrival, among them:

- creates the patient data base file if one does not already exist

- prefetches before archived studies to review stations for comparison

- on arrival, the RIS checks the patient in

- the radiology technician enters the patient name and ID in scanner (other information, if required by the scanner, can be pulled from the RIS avoiding dual data entry).

- the scanner pushes images to the local archive

- the radiologist pulls images from local archive for interpretation on review station

- the RIS attaches the verified radiologist's report to the patient's file

- images are marked "Interpreted," sent to the longterm archive, and are available to external HIS terminais.

- other clinicians pull studies for review via HIS terminals on ward.

Fig 3 shows the key elements involved if a patient is scheduled for an magnetic resonance (MR) exam and assumes that once the patient study resides on the longterm PACS, it has been interpreted and released for use to

The Radiology Department

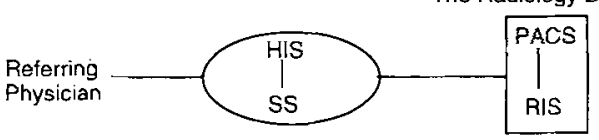

Fig 2. The data flow resulting from a physician for secretaryl using a HIS terminal to request a radiological exam for a patient.

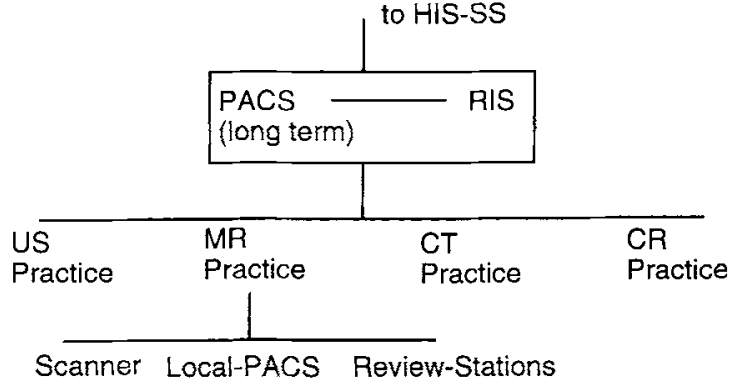

Fig 3. The internal data flow in the radiology department needed to move an MR image to the public PACS, which can be seen by the HIS network.

other services. Of course this description is only one possible scenario. For instance, some departments may not have a local PACS for each practice.

Once patient images are stored, healthcare providers are the principal agents that initiate new data flow. Radiology staff may view images from the local PACS or the long-term archive. The transaction is initiated at a radiology review station, and the data flow is completely intradepartmental. In the event that a clinician from another service wishes to see patient images, a more complex interaction occurs. First, we assume that the HIS terminals have the ability to display acceptable quality graphic images. Then a HIS user calls up the patient's EMR, selects the desired radiological exam, and the images are sent via the path shown in Fig 4. These transactions, and the amount of image information generated each day by each radiology modality, are summarized in the spreadsheet "Workload" section.

Performance. Performance issues involve parameters such as scanner throughput per hour, records fetch time (both within and outside of the Radiology department), image viewing conditions, and archival requirements. By quantifying the throughput of equipment and personnel, and comparing these figures with the workload, one can estimate hardware and staffing needs.

For the film based department, we use the maximum film processor throughput to compute the number of film processors and darkrooms required. For staffing needs (dark room and film records technologists, archivists, transcriptionists, secretaries, etc) we use empirical ratios (person/tasks ${ }^{*}$ day) observed at a large medical institution.

For the EMR based department, film processors and lightboxes are replaced with computed radiography (CR) units, computers, and networks. Because performance (and cost estimates) in the EMR environment will depend on this hardware ensemble, the question naturally arises as to how many computers are required, of what type, running across

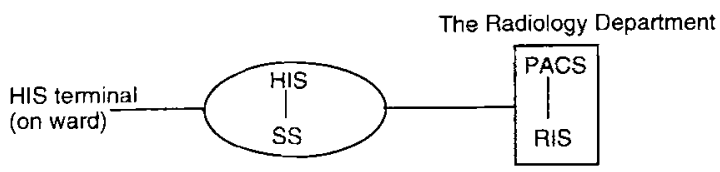

Fig 4. The data flow that occurs when radiology images are requested on a floor HIS terminal. 
what kind of networks and how do they communicate? We address these questions in reverse order.

If the communication protocols used between various information systems are portable, the type of computer used is largely irrelevant, assuming basic performance criteria are met. Data base requests between networked computers have often been communicated via the structured query language (SQL). A user sitting at a SQL client (a HIS terminal, for instance) would ask for lab results by sending a SQL request to the SQL speaking data base server in the lab department. Currently, Health Level 7 (HL7) is gradually replacing SQL in healthcare data bases because it is tailored for medical data. Radiology departments are also implementing Digital Image Communication in Medicine (DICOM) as both a file format, and a communication protocol to manage PACS images. ${ }^{5}$ Hence, it is possible that a HIS request to the RIS for patient images could be made in HL7, but the actual image transfer could be made in DICOM.

Assuming that the HIS, PACS, and RIS are speaking the same language(s), the next question is over what are they speaking? There are many possible answers depending on the distance involved, desired performance, and cost. For local area networks (LANs), the most popular network protocols are FDDI, Ethernet and Token Ring (with wireless variations on all). For longer distances there are: standard phone lines, integrated services digital network (ISDN), special leased lines, satellite, microwave, and even a new device called the "Cable Modem" that sends computer data over cable TV lines. Table 1 is a useful guide. ${ }^{6}$

Generally, greater bandwidth commands a higher price. However, for new LAN installations one can hedge by using Category 5 twisted pair cabling. Ethernet, Ethernet 2, and Token Ring will all run on Cat. 5 and upgrades to the system need only be done at the computer and router level, preserving the wiring investment. ${ }^{6}$

On the question of computers, different parts of the HIS-RIS-PACS chain perform different duties, so there is no need to have one hardware platform throughout. In fact,

Table 1. LAN and WAN Media

\begin{tabular}{|c|c|c|c|c|}
\hline Name & Media & Bandwidth & $\begin{array}{l}\text { Cost/ } \\
\text { Month }\end{array}$ & $\begin{array}{c}\text { Max. } \\
\text { Segment }\end{array}$ \\
\hline Ethernet & $\begin{array}{l}\text { coax, } \\
\text { twisted } \\
\text { pair }\end{array}$ & $10 \mathrm{Mbps}$ & NA & $100-200 \mathrm{~m}$ \\
\hline Ethernet 2 & $\begin{array}{l}\text { coax, } \\
\text { twisted } \\
\text { pair }\end{array}$ & $100+$ Mbps & NA & $50-100 \mathrm{~m}$ \\
\hline Token ring & $\begin{array}{l}\text { coax, } \\
\text { twisted } \\
\text { pair }\end{array}$ & 4, $16 \mathrm{Mbps}$ & NA & $100 \mathrm{~m}$ \\
\hline FDDI & fiber & $100 \mathrm{Mbps}$ & NA & $50 \mathrm{~km}$ \\
\hline $\begin{array}{c}\text { Standard } \\
\text { phone }\end{array}$ & twisted pair & $30 \mathrm{kbps}$ & $\$ 20$ & NA \\
\hline ATM & twisted pair & $100,155 \mathrm{Mbs}$ & $?$ & NA \\
\hline ISDN & twisted pair & $56 \mathrm{kbps}$ & $\$ 50$ & NA \\
\hline $\mathrm{T} 1$ & twisted pair & $1.5 \mathrm{Mbps}$ & $\$ 350$ & NA \\
\hline T3 & twisted pair & 44.7 Mbps & $\$ 2,500$ & NA \\
\hline Cable modem & $\operatorname{coax}$ & $10 \mathrm{Mbps}$ & $?$ & NA \\
\hline
\end{tabular}

A summary of LAN and wide area networks (WAN) costs and performance.
Table 2. Display Specifications by Practice

\begin{tabular}{lllcc}
\hline \multicolumn{1}{c}{ Practice } & Pixel Size & Resolution & Gray Scale & Luminosity \\
\hline Chest & $.2 \mathrm{~mm}$ & $2.5 \mathrm{lp} / \mathrm{mm}$ & $12 \mathrm{bit}$ & $180 \mathrm{fL}$ \\
Skeletal & .05 & 10 & 12 & $180 \mathrm{fL}$ \\
Gl & .4 & 1.25 & 12 & $180 \mathrm{fL}$ \\
CT \& MRI & .8 & .625 & 12 & $180 \mathrm{fL}$ \\
US/NucMed & .8 & .625 & 8 & $75 \mathrm{fL}$ \\
Mammo & 0.02 & 20 & $10 \sim 12$ & high \\
\hline
\end{tabular}

Recommended resolution and luminance criteria for radiology review stations by practice.

to do so would be financially unwise as the graphic performance required of a radiology review station would be wasted in a RIS terminal.

Because HIS terminals will be liberally distributed throughout the institution, the unit costs must be carefully considered. However, because the HIS terminal must be capable of displaying text, data plots, and images, a graphical user interface (GUI) is required. As HIS terminals will not be used for primary diagnosis, display requirements can probably be met with $1,000 \times 800$ pixel displays. This puts the HIS technical requirements in the arena of conventional desktop computers such as the Macintosh or MS-Windows equipped PCs. Disk requirements are not great because the primary purpose of a HIS terminal is simply to display information contained on departmental information systems. Aside from the basic video resolution, the only other needs are sufficient RAM and network bandwidth to achieve acceptable image retrieval and painting speeds.

A RIS terminal's primary role is dealing with textual information: patient demographics, links to the PACS images associated with the patient, and radiology reports. None of these tasks would require a GUI, but the majority of RIS vendors use one because of the enhanced user productivity. ${ }^{4}$ Additional efficiencies can be gained if RIS terminals are located near the radiology review stations and support report editing, either manually via typing, or in conjunction with voice recognition systems that allow real time dictation. ${ }^{7}$ Disk, RAM, and networking requirements are similar to HIS terminals, but even lower resolution monitors could be used, furthering lowering unit costs.

In purchasing a radiology review workstation, several factors (monitor resolution, luminance, image retrieval, and paint speed) are critical in affecting performance for the radiologist. Furthermore, resolution and luminance needs vary from practice to practice. Table 2 is a useful reference. 8.9

In addition to basic display requirements, each practice may have specific features that are useful to them. Hence, each practice may prefer its own review platform: perhaps Macintosh based software for ultrasound, MS-Windows tools for mammography, and UNIX machines to view computed tomography (CT) and MR images. As long as the PACS archive and review stations are (truly) DICOM compliant, it should not matter what mix of review stations the department uses. Of course, strict attention to the vendor's DICOM conformance statement and testing is still advised.

At this point it is also convenient to discuss teleradiology, which can be considered a longer distance application of radiology review stations, and the features desired in one 
setting will be equally useful in the other. Some vendors employ a point to point scheme, which requires a modem equipped sending computer to directly dial the local teleradiology system. ${ }^{9}$ Although inexpensive, this method is inflexible in that if the radiologist is at home or any other arbitrary location, the person sending the images must find out where the radiologist is and hope there is a nearby computer that can receive the images instead. A better approach is to have a dial-in facility at the central PACS archive, which is always available for incoming images (see Fig 5). Radiologists can then retrieve studies from any convenient location, perhaps even from home while on call.

The security concerns that arise from opening up the PACS to external phone line access can be addressed in a number of ways. Image senders would only need access to a phone number that directs them to an account that only allows incoming images. On a UNIX based PACS archive, a user login and password could be required before access to the archive occurred. Finally, the source of images could be authenticated by using public key cryptographic methods, which could then be double checked against the claimed username and password. Image reviewers could be given another phone number to an account that only allows image viewing. The additional steps outlined for image senders could also be applied in this instance to authenticate the identity of the image reviewer.

Costs. Costs are broken down into capital and annual "maintenance" classes. The capital budgets compute the number of units needed for each task based on defined workload and throughput per unit per hour. Annual costs are broken down by personnel and hardware. Personnel costs include salaries and benefits, whereas hardware includes consumables (computer disks and film), waste disposal (for photographic chemistry), and service contracts. The spreadsheet concludes with a summary of the total budget, and the fractions spent on capital verses annual maintenance costs.

\section{DISCUSSION}

Obviously, no outsider can begin to quantify the workload, performance needs, and costs for a specific institution. Patient loads can vary from month to month, networking costs vary from location to location, and the list price for any medical equipment is almost never the price paid. Therefore, in the appendices we provide spreadsheets that can be modified to perform

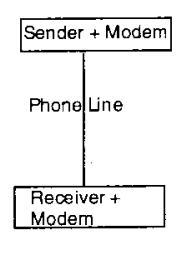

Point to Point vs.

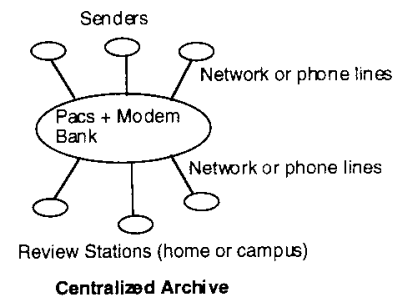

Fig 5. A contrast of point-to-point versus centralized archive approaches towards teleradiology. custom cost comparisons, using numbers relevant to a specific institution.

To show the utility of the spreadsheets, we simulate three institutions with varying case loads to determine when costs favor film, favor digital, and when the two models break even. This is accomplished by computing the differential costs needed to equip and operate an all digital versus all film-based radiology department for 1 year. A number of common take home points emerge. First, the vast majority of computer network bandwidth is consumed by images, much of which originates in Radiology. It is incumbent on the Radiology department to keep the IS department fully informed of planned needs. By comparison, the bandwidth used in text based information transfers is negligible. In fact it is probably not overstating the case to say that the institutional network should be planned jointly by Radiology and IS.

Second, a point not made explicit in this analysis, but very clearly by others, is the impact of network speed on total HIS-RIS-PACS costs. ${ }^{2}$ Put simply, the faster the network, the less storage capacity is required per workstation and the cost advantage scales linearly with the number of workstations.

Third, a large proportion of any cost savings to be had by moving to an EMR environment are realized at the personnel level. Trying to make a case for digital imaging based on saved film handling costs alone is difficult. Moreover, the cost advantage grows as more automated integration occurs (ie, between the HIS and RIS). To expand on this point, we estimate the personnel needs for the film department on empirical ratios observed in a large institution. The personnel estimates for the digital department are more conjectural. For instance we assumed:

- Only $20 \%$ of scheduling positions will be cut. Savings assumed to be gained from greater throughput via SS-RIS (and other departmental IS) negotiations.

- $80 \%$ of hospital records positions will be cut because care providers will be able to access the patient record from floor HIS stations.

- Radiology secretary positions will remain unchanged.

- $80 \%$ of Radiology transcriptionists will be 
cut assuming widespread use of voice recognition systems.

- Dark room, film, and archiving positions are absent in a filmless department.

Naturally, these assumptions are open to discussion. Not all practices will have all these positions and some institutions may combine tasks under one job title. In these cases, the number of full time equivalents (FTEs) for a specific job title may be set to zero or have its value transferred to another job title. Given the staffing assumptions previously discussed, Table 3 and Fig 6 summarize the costs for 3 institutions with 25, 50, and 105,000 studies per year. Breakeven occurs near 50,000 (actually 48,200) studies per year, which is in remarkably good agreement with earlier predictions. ${ }^{2}$ Interestingly, digital capital costs are only loosely coupled with case load whereas film annual and capital costs reflect a much stronger dependence.

There are several more intangible cost savings from the EMR environment that we have not attempted to model here. One benefit is "load balancing" by performing some procedures at satellite clinics and transmitting the images to the central reading area. This effect can minimize new scanner purchases by limiting overcrowding at the central imaging site. Overtime costs may also be reduced if technologists can leave work on-time because of diminished turn around times from patient scan to radiologist dismissal of the patient..$^{10}$ Also, the greater tolerance of computed radiography (CR) to over/under exposure may lower repeat rates, further diminishing technologist overtime or increasing patient throughput. Although these effect are certainly important, they are difficult to predict.

Finally, to compare digital and film-based departments, we assume that the study load is held constant in both scenarios. This would almost certainly not be true. Some studies indicate that overall radiologist productivity may increase $10 \%$ to $30 \%$ in the filmless environment (despite spending more time per case) because of other gains. ${ }^{11}$ Recent user interface developments could also reduce the time spent interpreting each study. Enhanced interfaces (incorporating modality sensitive automatic window and level and other features) should allow review station users to become more efficient, and scanner throughput can be increased by reducing technician time on nonimaging activities (assuming more patients are available to be scanned).

\section{CONCLUSION}

Digital radiology systems offer many nonfinancial advantages, but cost justification is frequently cited as the principal motivation for adopting them. By performing the cost analysis outlined in this report, one is compelled to make explicit assumptions and justify them before making the statement, "The digital department will save money."

Through the use of our spreadsheets, we believe anyone can perform their own cost analysis and decide whether or not to embrace the new technology. Also, by considering the issues involved, one gains a deep understanding of the EMR environment and the components in the HIS-RIS-PACS chain. By tailoring the spreadsheets to fit a given practice and budget, one arrives at a deep understanding of the required specifications for each component.

\section{APPENDIX 1: DIGITAL DEPARTMENT COST ANALYSIS}

This Appendix and the next describe how to use the spreadsheets to model a specific practice. The spreadsheet file (for Microsoft Excel

Table 3. Cost Summaries for Three Institutions

\begin{tabular}{lllllll}
\hline & $25 \mathrm{~K}$ Dig. & $25 \mathrm{~K} \mathrm{Film}$ & $50 \mathrm{~K}$ Dig. & 50K Film & 105K Dig. & $105 \mathrm{~K}$ Film \\
\hline Capital & $\$ 1.7 \mathrm{M}$ & $\$ 0.63 \mathrm{M}$ & $\$ 1.7 \mathrm{M}$ & $\$ 0.78 \mathrm{M}$ & $\$ 1.8 \mathrm{M}$ & $\$ 1.2 \mathrm{M}$ \\
A-Hw & $\$ 0.11 \mathrm{M}$ & $\$ 0.068 \mathrm{M}$ & $\$ 0.12 \mathrm{M}$ & $\$ 0.13 \mathrm{M}$ & $\$ 0.13 \mathrm{M}$ & $\$ 0.3 \mathrm{M}$ \\
A-Stff & $\$ 0.46 \mathrm{M}$ & $\$ 1.0 \mathrm{M}$ & $\$ 0.80 \mathrm{M}$ & $\$ 1.7 \mathrm{M}$ & $\$ 1.6 \mathrm{M}$ & $\$ 3.5 \mathrm{M}$ \\
Total & $\$ 2.3 \mathrm{M}$ & $\$ 1.7 \mathrm{M}$ & $\$ 2.6 \mathrm{M}$ & $\$ 2.6 \mathrm{M}$ & $\$ 3.5 \mathrm{M}$ & $\$ 5.0 \mathrm{M}$ \\
\hline
\end{tabular}

An output summary from the spreadsheet for 25,50 , and 105,000 diagnostic procedures per year. Only the number of cases were changed, other costs were computed from the spreadsheet's defaults. Note that annual staffing (A-Stff) costs increase linearly as a function of case load for both the film and digital departments. However, annual hardware (A-HW) costs are largely flat for the digital department, whereas the film department's costs track the case load. 

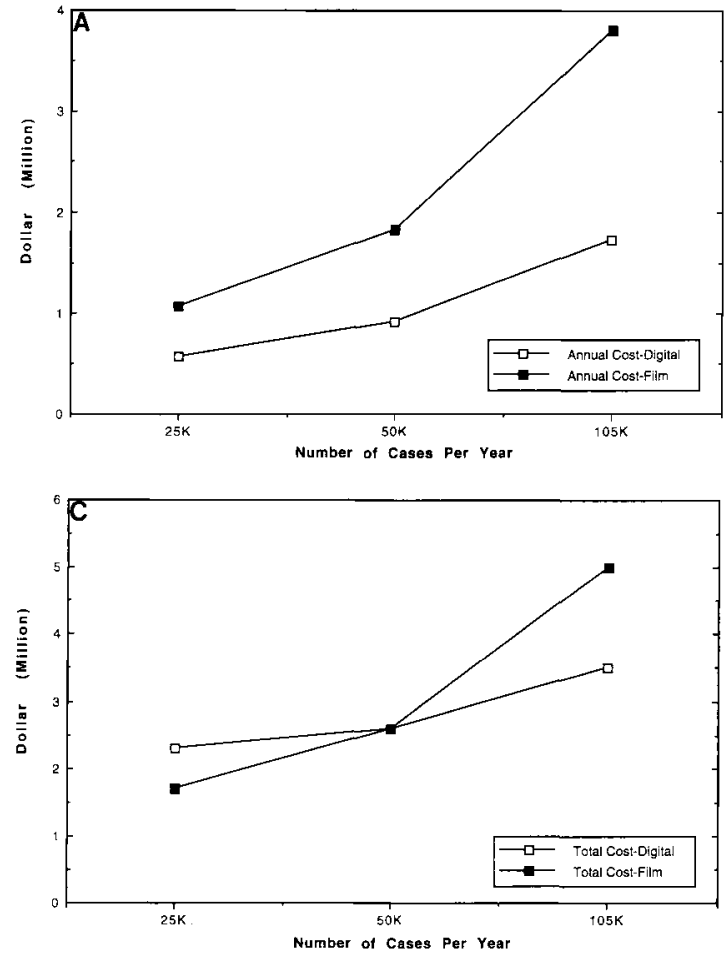

V4 or later) can be found in both Macintosh and PC formats at the anonymous ftp site (be sure to use a "binary" transfer and that your ftp tool understands files ending with xls):

ri-exp.beaumont.edu/pub/diag/Dos/ pac-cost.xls or ri-exp.beaumont.edu/pub/ $\mathrm{diag} / \mathrm{Mac} / \mathrm{pac}-$ cost.xls

As retrieved the file models a moderate sized (500 bed) hospital, which performs about 100,000 diagnostic procedures per year. The spreadsheet actually contains both the digital and film-based models on the same page-the digital analysis runs vertically down the left side and the film analysis does likewise down the right. Analogous sections (ie, annual hardware costs) lie side by side to facilitate comparing similar calculation stages in the two scenarios.

In this Appendix, we will describe the entries for the digital analysis. The first point to notice is that most of the cells are locked. This is because many of them contain formulas that reference values in other cells (to see the formula, hilight the cell of interest and view the message area under Excel's menu). We have left unlocked all the cells that should be required to enter custom data. However, if you

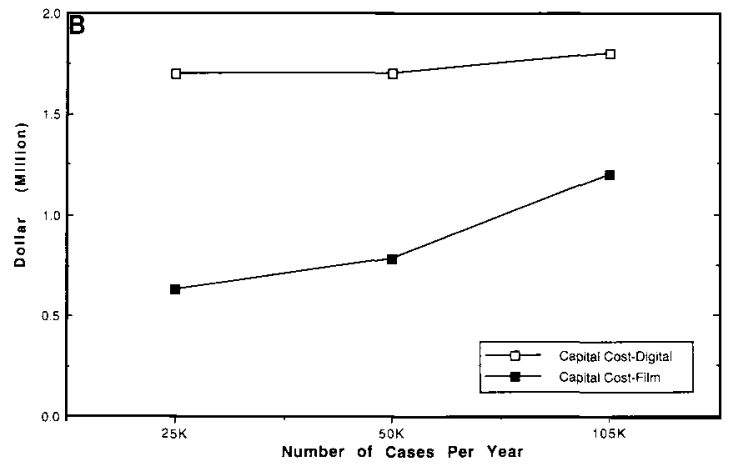

Fig 6. A series of plots showing the annual (A), capital (B), and total $(C)$ costs for maintaining a digital or film-based radiology department for one year at various case loads. Note that break even occurs near 50,000 cases per year. Also, the film-based costs are much more strongly dependent on case load than are digital costs. Hence the cost gap increases with increasing case load.

find that you want to modify the formula in a cell, simply "Unprotect" the spreadsheet.

Workload. We start by quantifying the workload for each subpractice within the department. The "Number of Images per Study," image dimensions, and "Studies per Day" fields can all be modified. The other locked fields perform calculations on these values.

The network load tallies the number of text and image transactions associated with a typical study. "Ave_HIS_pull/day" refers to the average number of times that a given study may be pulled out of the archive and viewed on a ward HIS station. If this number is increased, the "Average Network MB/study" value also increases which raises the average megabits per second (Mbps) load on the institutional network. The "Ave Mbps" value is an important indicator of the average network load that can be expected. It should be compared with the table of network speeds in the Materials and Methods section. Some caveats should be mentioned at this point:

- Image prefetch is counted as a daytime transaction. An intelligent algorithm would perform this overnight (except for ad hoc cases). 
- We weight HIS image transactions equal to PACS transactions within Radiology. In fact, HIS images would probably have reduced resolution and hence be smaller.

These points tend to overestimate the network traffic. However, these are only two components of the total number of image transactions and if required, they can be weighted less in summing the "Image_Transactions/study."

Performance. Selecting radiology review, HIS and RIS computers is a deep topic that involves features, interface, and software compatibility issues best left to another report. The primary purpose of the "Workstation" chart in the Performance section is to tally up components once a vendor and model are selected. Issues of archive query and study retrieval speed are dominated by network performance.

On-line archive capacity includes mini-archives for each modality and a long-term archive. If a given modality does not have its own archive, the associated "Required Day" field can be set to zero. In this example, we have assumed that the mini-archives are single platter optical drives whereas the long-term archive is assumed to be an optical juke box.

Network performance is computed by dividing the average study size by the seconds that one is willing to wait for the study to arrive. This is somewhat overkill, because often a user is content to have the first image arrive within a certain time whereas the remaining images arrive in the background. Also, at this point one has to consider the background network traffic. In a simple one segment network, the "Ave Mbps" computed in the "Workload" section has to be added to the bandwidth required for-say a fast CT study retrieval. When all those bandwidth requirements are added in, the shortcomings of standard Ethernet or Token Ring become quickly apparent. Of course, one normally "subnets" network segments by floor or department so that traffic is not all combined on one line. However, even subnetting may be insufficient for CR or large CT and MR studies on standard Ethernet. Ethernet II or FDDI, combined with lossless compression, can yield rates approaching $300 \mathrm{Mbps}$. For our cost analysis, an Ethernet II system is assumed for the hospital LAN.A 3:1 lossless compression method reduces network loading for archive to review station transfers, but full image data is assumed on the archive. For teleradiology, ISDN lines with $50 \sim 60: 1$ wavelet compression is assumed.

A chart for CR units is included, although in some situations this may not be a differential unit because many clinics use $\mathrm{CR}$ with film. One could set the CR unit price to zero in this case, but we include it on the premise that the film-based department used screen-film. We do not include the cost of film digitizers because we assume digital acquisition throughout the institution. Also, we do not model any hard-copy capability. However, it would be trivial to include the costs of dry film technology laser cameras if some hard-copy capability is desired.

Capital costs. The unit costs of all workstations is meant to include both the value of the hardware and the software licensing fee amortized per unit. The number of teleradiology workstations (which are assumed to be at radiologist's homes) is linked to the number of radiologists (which is defined in the film analysis "Performance" section under "lightboxes"). Both mini-archives and the long-term optical archive are presumed to have minimal heating, ventilation, and air conditioning special requirements.

Network cards are summed from the total number of workstations. Hub and router costs are based on Ethernet II equipment and the number of units is derived from the ports/hub and routers/floor entries in cells G27 and G28.

Annual costs. Service contract fees are based on a percentage of the unit cost. The percentage is defined in cell G31. Teleradiolgy line fees are based on monthly costs for ISDN service.

Numbers of staff positions are based on percentage reductions from the staff needs seen in the analogous chart under the film analysis. The reduction value is in the formula for each cell.

\section{APPENDIX 2: FILM DEPARTMENT COST ANALYSIS}

The model for the film-based department is similar to the digital one. To make comparisons, the "Studies/day" fields should be kept equal for the two workload sections, although the spreadsheet does not require this.

Workload. "Images/study," "Images/film," and "Study/day" fields can be modified. The 
"Films/day" field assumes a single duplicate for each film for extra departmental referrals. The number of human transactions per study is tallied and later used to compute the required number of film records positions as a function of workload.

Several staffing ratios are defined such as the number of radiologists per Radiology secretary. These values are arrived at empirically. For practices that combine secretarial and transcription duties in one position, these ratios may have to be altered and the staffing charts in the "Annual Costs" section modified to combine the job titles.

The "Work days per year" and "Hours per day" entries are referenced by cells in both the film and digital analyses to compute workloads and archive requirements, but can be modified. The "Studies_Scheduled/patient" field is used to compute scheduling staff requirements based on case load. Film and chemistry costs are defined to compute the annual costs of maintaining the film processors, and disposing of the waste.

Performance. In computing film processor requirements, we presume that a manual processor is used by the plain film, nuclear medicine and fleuro modalities (fleuro cine and mammo processors are not considered differential items because they will probably continue to be used in the digital department for some time). CT, $\mathrm{MR}$, and US modalities are assigned to a networked laser camera. For this example, we have not included a multiloader processor, but the cost charts allow for this. If a multiloader is substituted, remember to compute the number of units required in the capital cost chart by summing the films assigned to the multiloader and dividing by the multiloader's throughput.

The lightbox chart is fairly self-explanatory except for two implicit details. The number of modality offices is also taken to correspond to the number of radiologists (one radiologist per office). The number of reading areas on the ward is the product of the number of HIS stations per floor with the number of floors (in other words, we assume a one-to-one replacement of existing ward lightboxes with HIS stations).

Archive space is computed by summing all studies generated per year and assuming that each study folder has the dimensions listed. The resulting value is the additional archive volume needed each year. Dividing by 8 (standard ceiling height in feet) yields the additional archive area which must be leased in the "Annual" costs section.

Staffing needs per task are estimated from empirical ratios observed for the named tasks in a large medical institution. For example, we observe that our facility uses 20 film archivists to retrieve and file 5,600 studies per day. Because these ratios may vary at other centers, these cells can be changed. The "Study/day" cells reference the earlier workload estimates to compute the required number of FTEs.

Capital costs. The "Unit Cost" fields may be modified for all charts in this section with the exception of the darkroom cost. This value is arrived at from a standard construction formula for darkrooms, which is based on square feet, plumbing, light tight doors, and other variables.

The required number of units is a locked cell, based on earlier defined film volume and throughput. The minimum number of film processors required is computed because we presume maximum usage. If convenience dictates that additional processors are required (ie, because of a great distance between some modalities) the additional number of processors must be added by hand to the "no. of units" field of the processor chart. The number of required darkrooms (and other dependents) will be updated automatically.

Annual costs. The first three rows of the hardware chart tally the chemistry costs needed to run each processor type for one year, and add the film costs that are associated with that processor type from the workload calculations. The chemistry and service contract fields can be modified while the other cells are protected. The remaining three rows allow the "Cost/unit" cell to be modified, whereas the number of units and total price are computed automatically.

The total number of chemistry waste units is found from summing the total fixer and developer costs, and dividing by the respective costs/ barrel. This yields the number of barrels (55 gallons) of fixer and developer. These figures are added and then multiplied by five (because the chemistry is diluted 5:1 with water) to arrive 
at the total number of barrels which must be disposed of.

The number of lightbox bulbs that must be replaced each year is assumed to be a fixed percentage ( $10 \%$ in our estimate) of the total number of installed bulbs.
The "Personnel" chart number of FTEs for each job title is based on earlier computations. Only the "Salary + Benefits" field is left unlocked. Again, to compare costs with the digital model, the costs should match the analogous table in the digital analysis.

\section{REFERENCES}

1. Stut JJ, Didden HW, DeValk JP, et al: Prediction and analysis of PACS performance with the simulation tool MIRACLES. Med Inf 13:349-359, 1988

2. Beard D, Parrish D, Stevenson D: A cost analysis of film image management and four PACS based on different network protocols. J Digit Imaging 3:108-118, 1990

3. Bengtson N, Meehan P: Simulation of patient flow in a picture archiving and communications system network. Int J Biomed Comput 23:137-153, 1988

4. Hayes E: Systems integration: Information needs soar in managed care. Diagn Imaging September: 24-27, 1995

5. Taira RK, Breant CM, Chan HM, et al: Architectural design and tools to support the transparent access to hospital information systems, radiology information systems and picture archiving and communication systems. J Digit Imaging 9:1-10, 1996
6. Enck J: LAN to WAN. New York, NY, McGraw Hill, 1995

7. Frank MS, Guse SL, Stern EJ, et al: Integrating a digital dictation system with a network database for creating and managing radiologic teaching files. Invest Radiol 29:652655,1994

8. Osteaux M, Van Den Broeck R, Temmerman Y, et al: Medical requirements for clinical integration. A Second Second Generation PACS Concept, New York, NY, Springer-Verlag, 1:23-43, 1992

9. Barnes GT, Morin RL, Staab EV: Teleradiology: Fundamental considerations and clinical applications. RadioGraphics 13:673-681, 1993

10. Cannavo MJ: In my opinion: Evaluating the PACS payback timetable. Diagn Imaging September: 13-16, 1995

11. Siegel EL, Diaconis JN, Pomerantz S, et al: Making filmless radiology work. J Digit Imaging 8:151-155, 1995 